\title{
Pensiero divergente e Didattica DELL'ITALIANO LS
}

PAOLO TORRESAN*

ABSTRACT: In questo saggio definiamo la natura del pensiero divergente e la sua relazione con un tratto importante per l'apprendimento delle lingue: la tolleranza all'ambiguità. In secondo luogo passiamo in rassegna alcune strategie che permettono di attivare il pensiero divergente nell'insegnamento dell'italiano: la formulazione di associazioni e di ipotesi, la moltiplicazione dei punti di vista, la trasformazione dell’input. PAROLE CHIAVE: creatività; didattica dell'italiano; unità didattica; circolarità.

RESUMO: Neste ensaio definimos a natureza do pensamento divergente e sua relação com um aspecto importante para a aprendizagem das línguas: a tolerância em relação à ambiguidade. Em segundo lugar, examinamos algumas estratégias que permitem ativar o pensamento divergente no ensino do italiano: a formulação di associações e hipóteses, a multiplicação dos pontos de vista, a transformação do input.

PALAVRAS-CHAVE: criatividade; didática da língua italiana; Unidade didática; circularidade. 
ABSTRACT: In this essay we define the features of divergent thinking and its relationship with an important factor in language learning: ambiguity tolerance. Secondly, we consider a number of strategies for teaching Italian: Hypothesis - and Association-making, Stimulating multiple points of view, and Input transformation.

KEY-WORDS: creativity; Italian language teaching; teaching unit; circularity. 


\section{Pensiero divergente e tolleranza all'ambiguità}

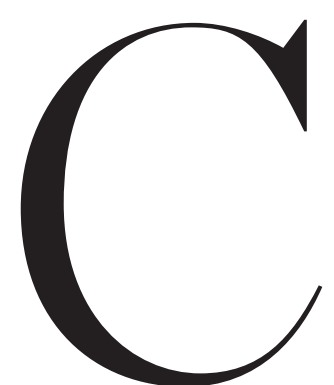

on il termine pensiero divergente intendiamo l'elaborazione di ipotesi, associazioni, interpretazioni lontane dai luoghi comuni.

Si tratta di una caratteristica propria delle personalità creative e prossima alla cosiddetta tolleranza all'ambiguità, ovvero la disponibilità a reggere la 'fatica' di un apprendimento complesso, come quello linguistico. In una comunicazione in lingua straniera molti sono i dati mancanti e le negoziazioni necessarie, c’è un grado di imprevedibilità e il rischio di fare brutta figura è sempre in agguato: se un soggetto non è disponibile ad affrontare lo stress legato alla gestione di queste variabili, difficilmente ha modo di progredire nello studio e con gran probabilità sviluppa un sistema di credenze e di atteggiamenti poco efficaci (EHRMAN, 1999).

Da un punto di vista metodologico contribuiscono a formare la tolleranza all'ambiguità pratiche 'rassicuranti' che rientrano nell'ottica dello scaffolding. La nozione di scaffolding venne formulata da Jerome Bruner (WOOD et al., 1976), e si riferisce all'impalcatura di legno delle case dei coloni americani. L'insegnante produce scaffolding ogniqualvolta aiuta lo studente a strutturare l'esperienza, fornendogli appunto un sostegno, una guida, un modello, delle regole. Così avviene durante: 
- la gradazione dei compiti e l'esposizione a rischi calibrati;

- la presentazione degli obiettivi a inizio lezione e la ricapitolazione di quanto fatto a fine lezione;

- la trasmissione di un atteggiamento sereno nei confronti dell'errore;

- le ricognizioni metacognitive, quando agli allievi è cioè permesso di fare il punto sui loro apprendimenti (cfr. DELMASTRO, 2010).

C'è da dire tuttavia che in molte scuole l'apprendimento si basa sulla ripetizione di contenuti (disciplinari) e di formule (linguistiche), senza che l'allievo sia messo a confronto con l'ambiguità; fatica, di conseguenza, a prodursi un atteggiamento sereno di fronte alla novità e a ciò che appare complesso.

Scrivono Dacey e Fiore (2000, p. 143-144):

Uno studio evolutivo sull'apertura mentale dei bambini mise a confronto le prestazioni di alunni di prima elementare con le loro prestazioni due anni dopo, quando si trovavano in terza. La ricerca trovò prove chiare del fatto che, benché i bambini in prima avvicinassero i problemi in maniera creativa, due anni dopo essi avevano perso significativamente la loro volontà di aprirsi a nuove idee. Il loro pensiero era diventato decisamente più rigido. Vi sono buoni motivi per credere che questa tendenza sia ancora più marcata nei bambini ansiosi.

Un altro studio, che prese in considerazione un ampio numero di classi elementari, trovò che il novanta per cento delle domande poste dagli insegnanti ammetteva un'unica risposta corretta.

Quando l'apprendimento è presentato puramente in termini di giusto o sbagliato, che motivazione potrà mai avere un bambino a esplorare nuove idee $\mathrm{e}$ prendersi qualche rischio in più?

Occorre quindi una giusta dose di scaffolding per produrre la tolleranza all'ambiguità, laddove, invece, le forme di guida e sostegno soffocano la libera iniziativa prevale la rigidità, che conduce ad una mancanza di stimolazione e a una passività nell'allievo.

Man mano che la competenza dell'allievo cresce e la richiesta di sostegno (scaffolding) per far fronte alla complessità viene meno (fading), sono proficuamente adottabili strategie di flessibilità cognitiva, in cui la complessità viene anzi 
'creata', l'ambiguità viene riprodotta e si sperimenta un'infrazione meditata delle regole (Fig. 1).

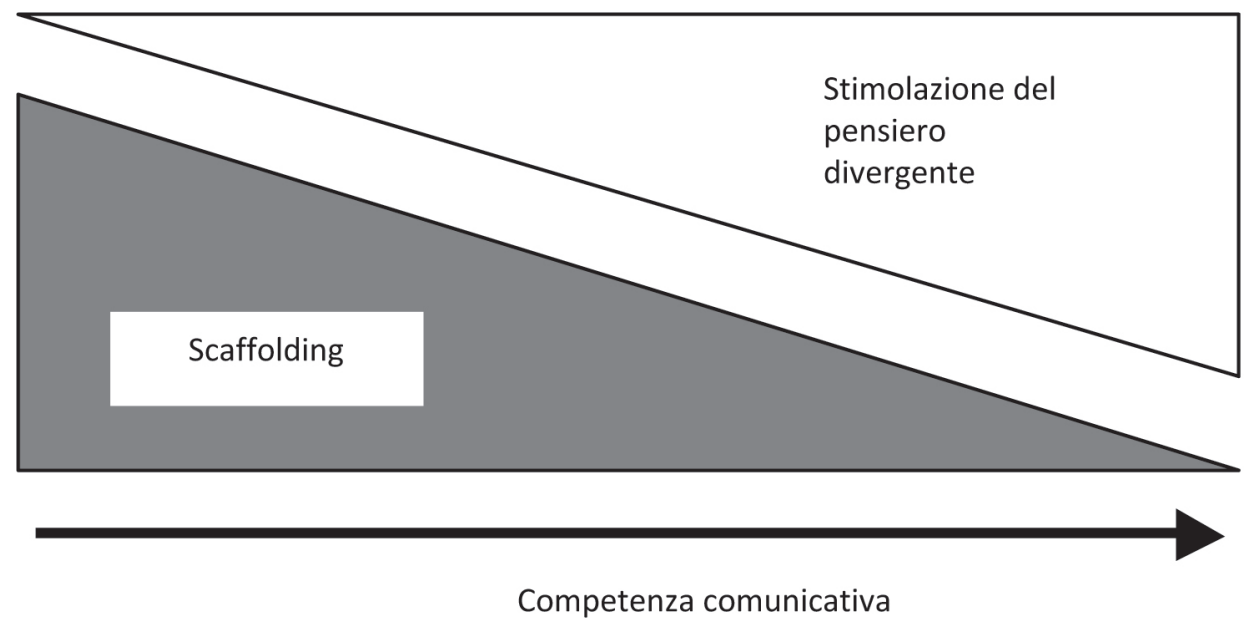

Figura 1

Rapporto tra scaffolding e pensiero divergente nell'evolversi della competenza comunicativa

Un atteggiamento esplorativo nei confronti della disciplina, capace di assumere rischi e di sperimentare, consolida una motivazione basata sul processo anziché sul risultato (cfr. CAON, 2007); nello specifico dell'educazione linguistica, ciò si rivela a vantaggio dello sviluppo, soprattutto, delle abilità comunicative (CASSANY, 1993).

In una ricognizione degli atteggiamenti e delle convinzioni rispetto allo scrivere in italiano, una nostra studentessa ventenne, Nadine, di origine austriaca, si espresse così:

[...] odio scrivere lettere più tosto vorrei parlarti. Il problema è che mai so cosa scrivere neanche nell' momenti importante. Gia nella scuola non mi ha piaciuto scrivere percepite ${ }^{1} \mathrm{o}$ sommari dei libri perciò mai sono stata brava.

$\mathrm{E}$ adesso ancora pegio perché devo spiegarmi in una lingua straniera e mi rendo sempre conto quanto mi manca dei vocabolari e quante errore faccio.

1 La studentessa voleva dire: "schede di analisi". Il suo testo viene riportato qui e nell'esempio che segue così come prodotto. 
La ragazza dichiarò perentoriamente "odio scrivere”: era un disagio che risaliva ai tempi di scuola, quando detestava i riassunti e le schede di analisi.

Al fine di preservare l'immagine di sé, Nadine, da adulta, attribuiva la causa dell'insuccesso al gusto personale ("non mi *ha piaciuto"). Tuttavia, è probabile si trattasse di una razionalizzazione: l'attività, da bambina, non le risultava gradita perché i passaggi necessari per portarla a termine non le erano mai stati chiariti (e forse non erano trasparenti nemmeno agli insegnanti che aveva). L'insuccesso, unito all' 'usura' degli strumenti, giocò, in tal caso, a sfavore della motivazione ad apprendere.

Il pensiero verticale (secondo la definizione di De Bono, 2004), ovvero il pensiero che procede secondo una direzione univoca di tipo logico-consequenziale (si pensi al fatto di compilare una scheda di analisi o di estrapolare i concetti fondamentali e di ordinarli, come avviene nel riassunto), spense la curiosità di Nadine, facendole associare la scrittura ad una sensazione di dispiacere. La paura di rischiare si cristallizzò, bloccando la crescita della Nadine-scrittrice. Il disagio ora, da adulta, a contatto con un codice che la giovane possiede solo in parte, l'italiano, affiora in tutta la sua evidenza.

Se gli insegnanti che ebbe ai tempi di scuola l'avessero spronata a tollerare la difficoltà, spiegandole, per esempio, che non si impara a scrivere tutto in un momento ma si procede per continue revisioni, e quindi, in seconda battuta, avessero valorizzato l'originalità della ragazza, forse, con gli anni, Nadine avrebbe maturato un altro sentire. Detto altrimenti, qualora chi avrebbe dovuto 'iniziarla' alla lingua scritta, l'avesse accompagnata alla scoperta delle strategie cognitive che sottostanno allo scrivere (scaffolding) e l'avesse stimolata a giocare con la scrittura (pensiero divergente), cogliendo le potenzialità espressive della lingua al di là della funzione referenziale, l'emozione di Nadine nei confronti dello scrivere, alle soglie dell'età adulta, avrebbe acquisito più i caratteri della sfida che quelli della mancata conferma ("mai sono stata brava").

Esiste difatti un circolo tale per cui la creatività si alimenta dei successi che uno sperimenta. Ł̀ un processo autotelico, in cui il fine coincide con il processo stesso: il soggetto creativo è immerso in una condizione di flusso, tale per cui l'investimento di energie non produce spossatezza ma si alimenta di emozioni positive: curiosità, coinvolgimento, soddisfazione (CSIKSZENTMIHALYI, 1993). 
Un primo passo verso la creatività consiste, dunque, nel valorizzare i tratti personali e unici dell'esperienza.

Basti un'indagine relativa alla visualizzazione che incorre durante l'ascolto di un racconto per vedere come ciascuno di noi realizza, inconsciamente, una sorta di film parallelo al testo ascoltato (cfr. PUCHTA et al., 2005; TORRESAN, 2009a); oppure chiedere agli studenti di segnare, sui margini del foglio, le associazioni personali che emergono durante la lettura per apprezzare la personalizzazione spontanea dei contenuti in fase di comprensione: il fatto ciò di riportarli alla propria esperienza.

In altre parole, la divergenza è una dimensione che ci appartiene, a tal punto che qualsiasi testo che leggiamo si 'mescola' con le esperienze pregresse: anzi, sono queste a conferirgli significato, valore.

Guardiamo, per esempio, le seguenti associazioni ai margini di un articolo (scaricato dal sito http://www.corriere.it/solferino/severgnini/07-01-25/01.spm) appuntate da Neil Wetzler, studente adulto di italiano, livello B2, di nazionalità statunitense. 
Le otto "o" per sfondare nel mondo di Beppe Severgnini

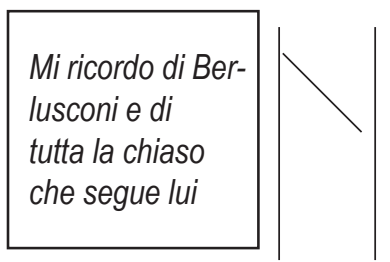

[...] Oggi il "Magazine" del "Corriere" parla degli italiani che hanno sfondato nel mondo (tanti, per fortuna). [...] Cerchiamo di capire se esiste, e qual è, il comun denominatore di queste storie di successo. Ci sono meccanismi virtuosi che in Italia possimo imparare ("imparare", non "importare": sul punto, ormai, non ho più illusioni). Propongo questo elenco, basato su otto $\mathrm{O}$.

Ci sono persone come queste dapertutto e io non li capisco
OMBELICO Un medico, un accademico, un cuoco o uno scrittore che non ha mai messo il naso fuori dall'Italia crede, inevitabilmente, che il suo ospedale, la sua università, la sua città o il suo editore siano l'ombelico del mondo. Uno scoop, signori: non è così!

ORGOGLIO Nel mondo conosciuto si può andar fieri del successo professionale. In Italia, il successo è qualcosa che bisogna farsi perdonare (e non sempre ci si riesce: Tamaro, Baricco e Muccino sono tre casi celebri; le aziende sono piene di casi meno noti).
Ho incontrato una ragazza italiana che aveva un bel apartamento a Venezia ma mi ha chiesto di non dire nessuno perché si vergogna 
ONORE Le belle storie internazionali sono quasi sempre trasparenti: talento, preparazione, lavoro, un'occasione. In Italia conta ancora troppo chi sei e chi conosci. Il caso dei concorsi universitari taroccati è clamoroso: ma neppure i migliori hanno il fegato d'intervenire. Preferiscono salvare la coscienza (e gli allievi) creando zone virtuose, e collezionare lauree honoris causa (sbaglio, professor Eco?)

Forse più italiani a quel età stanno ancora a casa quindi sentono "ancora ragazzi"
OSTACOLI Su 18.651 docenti di ruolo, solo 9 (pari allo $0.05 \%$ ) hanno meno di 35 anni; 5.647 (30,3\%) hanno più di 65 anni (inchiesta di Rizzo e Stella sul "Corriere"). Aggiungo: secondo un sondaggio Demos-Repubblica, il $39 \%$ degli italiani nella fascia 35-44 anni si definisce "giovane" e non "adulto". Come dire: talvolta le vittime sono consenzienti. In America, in Nordeuropa o in Russia non è così: a trent'anni si è uomini, non ragazzi. 


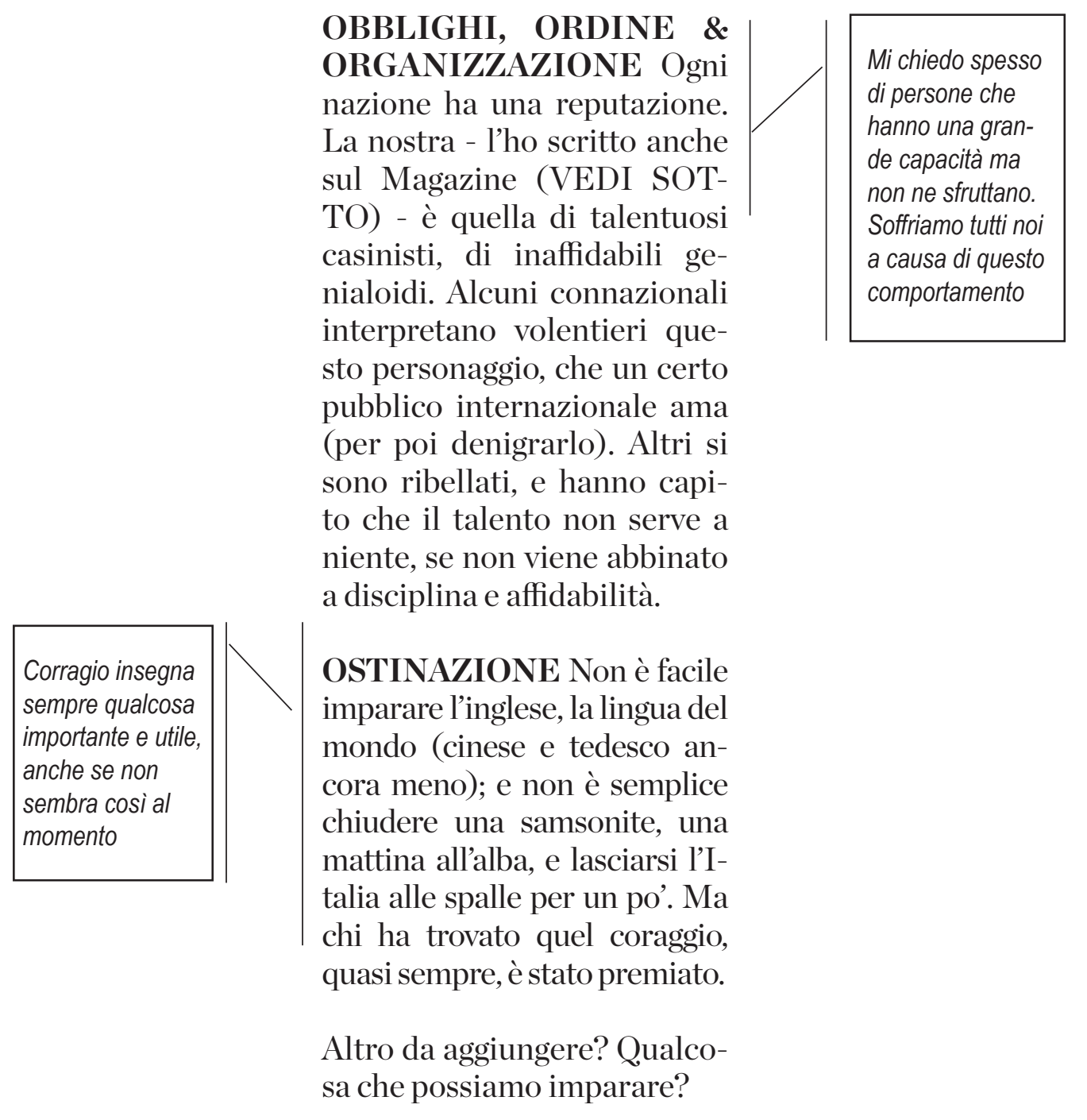

Queste associazioni non si aggiungono alla comprensione, ma ‘accendono’ la comprensione; fanno venir voglia all'allievo di tornare a leggere il testo, perché nel testo ha trovato qualcosa di sé. Ed è giocoforza che più uno legge, più ascolta. 
Lo stesso dicasi per il lessico: le parole che sentiamo non sono archiviate in compartimenti stagni ma sono collegate a sensazioni, immagini, emozioni, ciascuna delle quali 'accende' una rete di relazioni (cfr. STEVICK, 1986, p. 62; MORGAN, RINVOLUCRI, 2004²; MULLER, 2009).

È con grande stupore che, solitamente, studenti adulti scoprono che alcune immagini visualizzate e sensazioni provate durante il processo di apprendimento sono un patrimonio personale; per converso, è ingenua la convinzione che fa ritenere che tutti apprendano allo stesso modo.

$\mathrm{Ci}$ pare che il primo passo verso una didattica divergente nell'aula di lingue consista, in sostanza, nel prendere coscienza dei processi (associativi, in primis) che già a livello spontaneo ognuno mette in atto (RINVOLUCRI, 2009): rendersi conto, cioè, che la divergenza non è al di fuori, non appartiene ad altri, ma abita nel nostro modo di vedere il mondo e si manifesta ogni qualvolta, volentieri o nostro malgrado, siamo tenuti a cercare una soluzione personale ai problemi.

La divergenza è, quindi, un fattore intrinseco alla crescita; si accompagna al riconoscimento delle risorse individuali. "È una ricchezza interiore" - scrive Aznar (2009, p. 26; la traduzione è nostra) - "di cui ognuno è portatore. È un «tesoro» del mondo. È un processo ludico, liberatorio, un fattore di sviluppo personale che ha effetti terapeutici".

Nella storia delle lingue ci pare che il metodo strutturale e il metodo comunicativo rappresentino due istanze agli antipodi, in fatto di divergenza.

Da un lato, infatti, vi è una ripetizione meccanica di routine linguistiche, nella convinzione che l'alunno sia tabula rasa su cui l'insegnante incide il codice (linguistico, appunto, nel caso della lingua; ma potrebbe essere matematico, nel caso della matematica; musicale nell'ambito della musica; ecc. ecc.) e che l'errore sia da evitare perché genera un'abitudine che occorrerà sradicare poi.

Dall'altro lato, vi è un (prot)agonismo dello studente, invece, nella scoperta dei meccanismi che appartengono alla lingua (intesa non già come insieme di elementi discreti, bensì come sistema di sistemi); peraltro, si esprime tolleranza per l'errore, interpretato come momento fisiologico dell'apprendere. La mente di chi impara, in quest'ottica, non è tabula rasa, giacché, anche nell'attività che parrebbe più passiva, come la lettura di un testo, ognuno ci mette del suo.

Chomsky, all'epoca in cui segnò il sorpasso del metodo strutturale, chiamò in 
ballo la costruzione di sintagmi - da parte di bimbi che apprendono la lingua materna - assolutamente inspiegabili nell'ottica della tabula rasa. Se la mente fosse una tavoletta di cera, come si spiegherebbe, si chiese il celebre linguista, il fatto che un bambino formuli la frase "Daddy goed* out", senza che l'abbia mai udita pronunciare nel contesto anglofono in cui è cresciuto (CHOMSKY, 1959)? La mente di un bimbo è, invece, potente al punto tale da astrarre regole e generalizzarle. Un meccanismo meraviglioso presiede l'evolversi del linguaggio, attivo, peraltro, a prescindere dall'età e dal fatto che si impari la lingua madre o una lingua seconda.

Un'anziana di nostra conoscenza, che parla per gran parte del tempo in dialetto veneto, quando si trova in circostanze in cui deve parlare italiano, dà prova di errori dalla logica ferrea. Durante un pranzo nuziale chiese ai commensali se le potevano passare un kiwo*. Il figlio, presente, trasse (malauguratamente) motivo di sollazzo e condivise con gli invitati meno avveduti una bonaria commiserazione per l'errore della madre. Ma quanta intelligenza c'era in quell'errore (e quanta stupidità nel commento del figlio)! Se kiwi è il plurale, certamente kiwo* sarà il singolare, avrà inconsciamente pensato la donna.

Una collega di Salta (Argentina), Sandra Eugenia Acosta, registra gli errori dei bimbi che imparano italiano e ne studia, come fece a suo tempo Piaget, la logica. Quanta bellezza c’è, per esempio, nel lemma 'bambinera' espresso da un suo allievo, per indicare la maestra! L’allenamento alla divergenza è implicitamente una forma di riconoscimento dell'altro: della sua intelligenza, della sua immaginazione; in una parola: della sua personalità.

Siamo curiosi dell'altro (di ciò che dice, che fa, che racconta, persino degli errori che commette), ed è questo il modo in cui gli insegniamo ad apprezzare la curiosità.

Quando un bimbo spiega perché dice 'bambinera' al posto di 'maestra', dà ragione della sua associazione (e, mentre lo fa, pratica lingua!) e la condivide: la divergenza nasce nella relazione e si alimenta mediante la relazione.

Per illustrare ancora il concetto, consideriamo due modelli di addestramento di animali, descritti in Bateson (1984): uno operato dai comportamentisti (con una variante sadica), l'altro eseguito da una istruttrice divergente.

Nel primo caso un cane, mediante un serie di rinforzi positivi (cibo) e negativi (leggera scarica elettrica o anche solo mancanza di rinforzo positivo), apprende a 
distinguere un ellisse da un cerchio. L'addestramento fila liscio, fintanto che non insorgono situazioni ambigue. Tuttavia, se lo sperimentatore altera i dati e costringe a distinguere forme confuse, che nemmeno un occhio allenato riesce più a discriminare, il cane agisce arbitrariamente e, altrettanto arbitrariamente, riceve premi e punizioni. L'alterazione del contesto non viene accettata: l'animale si ribella e morde chi lo tiene al guinzaglio. È stato violato un patto, l'animale non si sente riconosciuto, perciò aggredisce.

Bateson cita, per contro, il caso dell'addestramento di una delfina (1984). Agli inizi, l'animale nuota liberamente nella vasca, osservato dall'istruttrice. Appena compie un gesto che l'istruttrice desidera sia fissato, il cetaceo riceve un rinforzo positivo (del cibo). Bastano tre rinforzi perché il gesto sia appreso. In un'occasione successiva, al ripetersi del gesto, succede qualcosa di sorprendente per l'animale: nonostante l'esibizione di quanto appreso, la delfina non viene premiata. Un qualsiasi altro gesto, pur se minimo, come il muovere la coda in segno di contrarietà, può essere però preso a modello dall'istruttrice e divenire oggetto di successivi rinforzi. L'errore, la divergenza, in questo caso, non sono puniti ma premiati: quello che l'istruttrice dimostra di rinforzare non è un comportamento predeterminato, ma una serie di comportamenti che prevedono la presa d'iniziativa dell'animale. La relazione (e quindi il riconoscimento) si sovraordina allo stimolo, è essa stessa lo stimolo.

\section{Strategie divergenti}

Immaginando che la divergenza sia la chioma di un albero, potremmo pensare dunque che lo scaffolding, e quindi ogni forma di guida che induce a una tolleranza/ gestione dell'ambiguità, equivalga all'humus e all'acqua presenti nel terreno. Allo stesso modo, è pari al sole, la cui luce consente di trasformare sostanze e acqua in linfa, la valorizzazione delle risorse individuali. Ebbene, quali sono allora gli agenti che, nel micro, consentono di tramutare la chimica in clorofilla, l'inorganico in organico? Fuori di metafora, quali sono le strategie mediante le quali un insegnante potenzia e sviluppa quella disinvoltura di idee di cui è capace lo spirito divergente?

Le rassegne di strategie volte a promuovere la creatività, anche nel solo ambito dell'educazione linguistica, sono parecchie; ci limitiamo a citare i recentissimi la- 
vori di Aznar (2009) e di Pugliese (2010), dai quali è possibile attingere una ricca bibliografia.

Tra le tante, abbiamo deciso di concentrarci sulle strategie che hanno una notevole malleabilità, consentono cioè di generare processi divergenti, anche quando sono nelle mani dell’insegnante che non coltiva di sé un'immagine formidabile in quanto a genio creativo. Tali strategie sono:

- connessioni e analogie

- ipotesi e previsioni;

- il moltiplicare i punti di vista;

- trasformare l'input.

Nei paragrafi che seguono illustriamo in dettaglio ciascuna di esse.

\subsection{Connessioni e analogie}

Una tra le pratiche più diffuse per l'elicitazione del lessico nell'aula di lingue è il brainstorming, in genere presentato mediante una mappa associativa. Durante la raccolta delle idee il docente è tenuto a dare libero spazio alla creatività: ogni ipotesi va accolta e rispettata, per quanto alcuni possano giudicarla impropria o bizzarra. Eventuali punti di divergenza possono casomai essere dibattuti e negoziati durante fasi successive. Più procede la raccolta, più l'asse si sposta verso la divergenza ed emergono quelle che Mednick chiama associazioni remote (1962). È l'ambito in cui si muovono i poeti e a cui non accede spontaneamente chi è portato ad assumere un atteggiamento dogmatico, teso a riprodurre le associazioni più consuete e condivise. L'associazione remota implica libertà dallo stimolo, la capacità di allargare la mappa delle soluzioni, abbracciando il più vasto campionario di possibilità (un buon test di flessibilità cognitiva è riportato in appendice).

Risorse che stimolano la produzione di associazioni sono anche i list poem, poesie composte da versi che si ripetono, come negli esempi riportati qui sotto, realizzati da studenti di livello C2. [I format dei componimenti sono attinti dalle seguen- 
ti raccolte: FAGIN 1991 (I); HOLMES, MOULTON 2001 (II; III); FORAPANI 2001 (IV)].

I. Rosso

Rosso come il vino

Rosso come il sangue

Rosso come le guance di chi si vergogna

Rosso come il tramonto

Rosso come Dio

Rosso come la parte più interna dell'uovo

Rosso come un pianto

Rosso come l'amore

II. Mi PIACE E NON MI PIACE

Mi piacciono le giornate di pioggia

Mi piacciono i tramonti sul mare

Mi piace il vento d'estate

Non mi piace passare la domenica così

III. CONTO ALLA ROVESCIA

Tre, gli alberi nel giardino

Due, le ore che mancano

Uno, il suono della campanella

IV. C'̀̀

C'è un uomo che legge

Cè una donna che piange

Cè una macchina nera

C'e tantissima gente

C’è un funerale 
Può stimolare la divergenza anche la costruzione di associazioni improbabili: lo studente, da solo o in gruppo, è tenuto a spiegare l'affinità tra termini che appartengono a campi semantici diversi, per esempio: telefono-fungo; albero-foto; librolampada.

Si può, inoltre, chiedere agli studenti di elencare gli usi più disparati che un oggetto può avere. Un libro, per dire, può essere utilizzato come:

- sottovaso o sottopentola;

- sonnifero (se noioso);

- arma letale contro una mosca;

- contenitore difarfalle e fiori secchi;

- combustibile;

- mezzo per dare stabilità a un mobile traballante, ecc.

Più in generale, al fine di far esercitare conoscenze disciplinari, agli allievi può essere richiesto di esplicitare i rapporti tra informazioni distinte; per esempio:

- A quali altre conoscenze all'interno di questa disciplina puoi collegare questo dato?

- A quali conoscenze che provengono da altre materie puoi collegare questo dato?

- Puoi collegare questo dato a situazioni di vita quotidiana?

- Come puoi esprimere questo concetto con un disegno? Con una musica? Con una serie di movimenti? Con una parola? Con un suono?

La capacità di operare delle analogie consente di vedere la realtà in maniera multiprospettica, evitando di ripetere nozioni stereotipate (GARDNER, 1995). ̀̀ un buon antidoto al pensiero rigido e permette di individualizzare l'apprendimento; l'allievo modella ciò che gli viene dato e lo fa suo.

Maria Assunta Simionato (2004) ha condotto esperienze di insegnamento della fonetica dell'italiano in cui bambini di prima elementare potevano rinominare alcuni gruppi fonetici con termini che, a loro parere, li rappresentavano meglio (tabella sottostante) 


\begin{tabular}{|c|c|}
\hline Denominazione scientifica & Denominazione prodotta dai bambini \\
\hline nasali & nasali \\
\hline occlusivi & scoppianti \\
\hline costrittivi & costrittali \\
\hline semiocclusivi & mezzoscoppianti \\
\hline vibranti & vibranti \\
\hline laterali & laterali \\
\hline
\end{tabular}

\section{Tabella 1}

Giuseppe Bevilacqua, docente di Educazione alla Voce presso l'Accademia Nazionale di Arte Drammatica 'Silvio D’Amico', chiede agli studenti che si accingono ad apprendere la fonetica dell'italiano di visualizzare ciascuna vocale con un colore e/o di abbinarla ad un suono presente in natura, quindi di immaginare la corrente d'aria emessa nel pronunciarla che arriva al lato opposto della stanza (cfr. BEVILACQUA, 2005). L'intenzione è quella di dare fisicità ad una nozione di per sé astratta, di renderla quanto mai ‘sensibile', nella consapevolezza che la memoria è un fenomeno distribuito; più grande è la varietà di codici mediante i quali un input viene appreso, maggiore è la probabilità che il ricordo sia duraturo (cfr. SABATANO, 2004).

L'insegnante stesso può modellare analogie tra la lingua e altri ambiti dell'esperienza. Per esempio, possiamo immaginare, come fa il collega Ciro Mazzotta, che i pronomi proclitici (definizione oscura per uno studente italiano) siano leggeri - isolati, tendono a 'salire verso l'alto' (es: mi perdoni) -, mentre quelli enclitici siano pesanti - formando un tutt'uno con il nome, acquistano peso specifico e 'scendono verso il basso' (es: perdonami).

Alcune analogie possono essere sollecitate da domande: A quale angolo della tua città abbineresti alcune parole che hai imparato oggi? A quale parte del corpo abbineresti, invece, il passato prossimo? Il futuro semplice?

Così facendo si produce una rappresentazione simbolica degli oggetti di apprendimento e, quindi, un aggancio di tipo analogico alle informazioni a cui si è stati esposti (non acquisite solo, dunque, per definizioni astratte). 
All'interno di questa prospettiva si leggano le molte proposte didattiche che fanno leva sull'espressività per illustrare un concetto (es.: realizza una scultura modellando il corpo dei tuoi compagni per rappresentare un certo tema, WESSELS, 1987), uno stato d'animo (es.: scegli un oggetto per rappresentare uno stato d'animo a fine corso, FERENCICH, TORRESAN, 2005; oppure: scegli una foto tra le molte che l'insegnante ti sottopone che meglio descriva quello che senti in questo momento), la propria personalità (cfr. la produzione di uno stemma personale in GRIFFITHS, KEOHANE, 2000) o quella dell'altro (es.: se il tuo compagno fosse una musica, che musica sarebbe? Se fosse un fiore, che fiore sarebbe?).

\subsection{Previsioni e ipotesi}

Mentre la connessione e l'analogia funzionano mediante libere associazioni, nella formulazione di ipotesi e di previsioni lo studente combina pensiero divergente e logica consequenziale, con ragionamenti del tipo se $x$, allora y oppure cosa succederebbe se?

Considereremo due ambiti in cui ipotesi e previsioni si possono impiegare in una lezione di lingua: la precomprensione e la verifica di quanto appreso in un contesto CLIL (didattica integrata di lingua e contenuti disciplinari).

Oltre che dal titolo e dalle parole chiave, in fase di precomprensione le ipotesi possono essere sollecitate da un'immagine, una musica, l'incipit del testo, spezzoni audio, fotogrammi, una rappresentazione mimata².

Questa fase può, del resto, esser dilatata a tal punto da prevedere una lunga serie di attività di produzione, prima di giungere all'incontro con il testo. Si tratta dei cosiddetti percorsi circolari, di cui abbiamo prodotto vari esempi (cfr. TORRESAN, 2009b, 2010).

Ora il percorso circolare si differenzia dal modello di Unità Didattica formulato a fine anni Settanta in seno alla scuola veneziana (FREDDI, 1979, ripreso in BALBONI, 1994; 2007). Il modello tradizionale, definito da Freddi, opera secondo una sequenza riassunta attraverso l'acronimo GAS:

- Globalità, la quale si compone di una breve precomprensione (PC) e di attività di comprensione $(\mathrm{C})$;

2 Una tecnica efficace per il recupero delle nozioni sociopragmatiche e di regole che afferiscono alla fonetica e alla morfosintassi è la ricostruzione di conversazione. L'insegnante mima lo scambio che avviene tra due personaggi, quindi invita gli studenti a formulare ipotesi circa il contenuto (cosa dice il primo personaggio al secondo?), aiutando gli studenti ad affinare le espressioni, fino a che giungono alla frase originale (cfr. D’ANGELO, 2006). 
- Analisi (riflessione sulla lingua): volta a dirigere l'attenzione su un elemento specifico (n), quindi siglata $A n$;

- Sintesi: reimpiego, mediante produzioni, più o meno controllate, della/e forma/e analizzata/e (n). Tale sintesi è rappresentata con la sigla $S n$.

Nella lezione circolare prevale, invece, una sequenza di tipo SGA:

- si parte da una Sintesi (S) di elementi analizzati in precedenza (a-m), orientata alla formulazione di ipotesi ( $\mathrm{PC}$, ovvero precomprensione);

- si giunge al testo da Comprendere (C);

- si procede con un'Analisi (A) relativa a un elemento specifico (n).

In sostanza, in un percorso circolare la precomprensione, anziché parentesi brevissima (costituita da esercitazioni lessicali, cfr. BALBONI, 1998) è un’attività di sintesi al tempo stesso; in essa l'allievo sviluppa e sostiene ipotesi frutto di una sua elaborazione e/o di una negoziazione con i compagni.
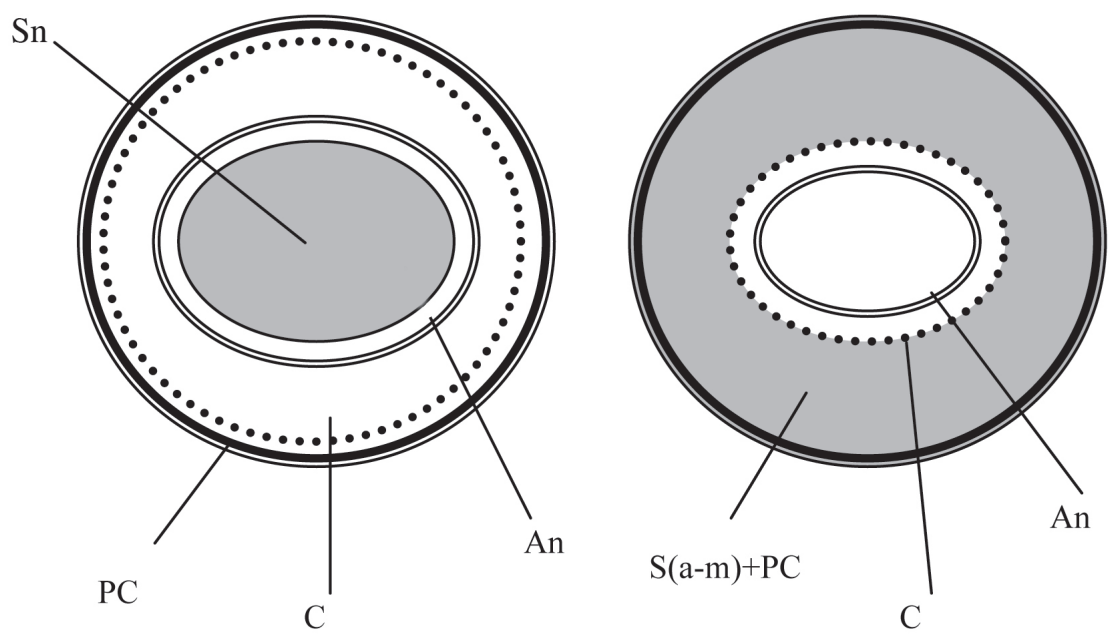

Figura 2. Unità Didattica GAS (che ruota attorno ad un elemento da analizzare n), a sinistra, ed Unità Circolare SGA (che ruota attorno ad un elemento da analizzare n), a destra ${ }^{\circ}$. 
Ebbene, siamo persuasi che i percorsi circolari siano una tra le palestre più attrezzate per allenare alla divergenza, un vero e proprio scaffolding alla flessibilità cognitiva, per via dell'ampio spazio dedicato alla formulazione di ipotesi e alla previsione in generale. Presentiamo qui di seguito un esempio di percorso circolare, ricco peraltro di strategie divergenti.

\section{FASE di SinTESI (precomprensione)}

Si chiede agli studenti di rispondere a una serie di domande: alcune, sono personalizzate, ovvero sollecitano il recupero di informazioni attinte dalla propria esperienza $(a-b)$, mentre altre costringono a formulare ipotesi $(c-e)$ :

a) Hai mai visto dei pesci? Dove?

b) Sei mai andato a pescare?

c) I pesci sono eterotermi: cosa vuol dire?

d) Un pesce può vivere nei mari del Polo Nord?

e) Tutti i pesci hanno le scaglie?

\section{FASE DI GLOBALITÀ (comprensione)}

Viene consegnato il testo. Durante la lettura ciascuno verifica la correttezza delle proprie ipotesi (il testo è tratto da Acquati et al. 2005, p. 93).

"I pesci sono eterotermi, in quanto la temperatura del loro corpo varia al variare di quella esterna.

Il fatto che la temperatura corporea dipenda da quella esterna potrebbe comportare dei problemi poiché, in un ambiente molto freddo, si abbasserebbe troppo. I pesci tuttavia non corrono questo rischio in quanto la temperatura dell'acqua non scende mai al di sotto di certi valori.

Il corpo dei pesci è, per lo più, rivestito da scaglie, strutture che si formano nello spessore della pelle e che possono avere diversa 
composizione. In alcuni casi, come nelle anguille, possono mancare”.

A lettura avvenuta, viene somministrata un'ulteriore batteria di domande, di diversa natura: referenziali (si riferiscono ad informazioni direttamente rinvenibili nel testo; $f$ ), inferenziali (si riferiscono ad informazioni 'nascoste', a cui si può attingere per induzione, collegando dati tra loro; $g$-h); personalizzate (mettono in relazione le informazioni lette con l'esperienza di ciascuno; $i$ ) divergenti (impongono di escogitare delle analogie; $j-k)$ :

f) Dove si formano le scaglie?

g) Quale funzione possono avere le scaglie?

h) Perché le anguille non hanno le scaglie?

i) Tu preferiresti essere un pesce con le scaglie o senza scaglie?

j) A cosa paragoneresti un'anguilla?

k) A cosa paragoneresti un pesce con le scaglie?

3. Fase di Analisi (riflessione sulla lingua)

Da ultimo, si può richiedere di praticare un’analisi personalizzata del lessico: ciascuno scrive il proprio nome al centro di un foglio, disegna cinque cerchi concentrici attorno, sceglie cinque parole tratte dal testo che gli suonano familiari, le dispone nei cerchi a seconda di quanto le senta vicine o distanti da sé. Infine, argomenta la propria scelta di fronte uno o più compagnis.

Veniamo al secondo ambito: la formulazione di ipotesi durante la verifica delle nozioni apprese in una classe in cui si pratica un insegnamento veicolare della lingua (per esempio, durante lo studio della storia).

Un test tradizionale è costituito in genere da domande aperte o chiuse (a scelta multipla o vero/falso), espressioni di un modello di apprendimento di tipo imitativo/ripetitivo. Domande di questo tipo potrebbero essere: 
- Quali sono le cause della Rivoluzione Francese e quale relazione intercorre tra loro?

- $\quad$ Tre di queste 4 opzioni rappresentano cause della Rivoluzione Francese: quali sono?

- la mancanza di cibo

- la personalità del re

- le idee illuministe

- l'eccessivo carico delle imposte

Alonso Tapia (2000) propone, in alternativa, un modello di rielaborazione di quanto appreso basato sulla formulazione di ipotesi. Egli assegna all'insegnante il compito di astrarre e di presentare alla classe le variabili che caratterizzano un certo fenomeno; in seconda istanza, all'apprendente viene chiesto di dire cosa succederebbe, se uno di questi fattori venisse a mancare.

- In quale paese è probabile che un gruppo sociale sia interessato a cambiare la situazione?

\begin{tabular}{|c|c|c|c|c|c|}
\hline & A & B & C & D & E \\
\hline $\begin{array}{c}\text { Transazioni } \\
\text { con l'estero }\end{array}$ & aumentano & diminuiscono & diminuiscono & aumentano & diminuiscono \\
\hline Prezzi & salgono & salgono & salgono & $\begin{array}{c}\text { rimangono } \\
\text { stabili }\end{array}$ & salgono \\
\hline $\begin{array}{c}\text { Imposte } \\
\text { Diritti e doveri } \\
\text { dei gruppi so- } \\
\text { ciali }\end{array}$ & aumentano & aumentano & $\begin{array}{c}\text { rimangono } \\
\text { stabili }\end{array}$ & aumentano & aumentano \\
\hline
\end{tabular}


Si tratta di un compito sfidante, dal momento che implica una rielaborazione profonda e fa sì che la lingua sia usata in modo autentico, per risolvere problemi.

La divergenza, in questo caso, si manifesta mediante un comportamento paradossale: si studia un fenomeno immaginando, per assurdo, cosa accadrebbe in situazioni analoghe, se elementi minimi venissero alterati. Il risultato indubbio è che l'allievo viene portato ad allargare la sua visione sui fenomeni: egli capirà che la rivoluzione francese offre, nelle vicende che l'hanno contraddistinta, un modello con cui leggere molti eventi della storia contemporanea. La divergenza contribuisce, così, a de-costruire un modo di pensare rigido e stereotipato.

\subsection{Moltiplicare i punti di vista}

Riconoscere che una stessa situazione può essere vista in due modi diversi è pure una caratteristica del pensiero divergente. A ciò possono contribuire:

- Compiti aperti, la cui esecuzione non obbliga a seguire un percorso definito. Pensiamo, per esempio, alla creazione di un fotoracconto, al montaggio di un videoclip (VIANELLO, 2001), alla rappresentazione di alcune sequenze mediante tableaux vivants;

- L'assunzione di identità fittizie, come indicato nella Suggestopedia, al fine di consentire allo studente un certo distacco rispetto alle imperfezioni delle proprie produzioni;

- Role play impostati su un conflitto comunicativo (due personaggi, all'insaputa l'uno dell'altro, perseguono scopi comunicativi differenti);

- Improvvisazioni nelle quali l'accento sia posto sulla pluralità delle motivazioni degli attori coinvolti, come avviene nel Process Drama (ampiamente utilizzato nella didattica dell'inglese, cfr. PIAZZOLI, 2009).

- La stesura di pro e contro di una certa situazione;

- La verbalizzazione dei pensieri dei soggetti ritratti in una foto;

- La riscrittura di un brano partendo da un punto di vista alternativo (la battaglia di Waterloo vista da un soldato morente; la selezione naturale vista da una specie in estinzione; la fiaba di Cappuccetto Rosso raccontata dal lupo); lo stesso valga per la descrizione doppia di un oggetto (per esempio: il 
proprio paese o la propria via visti da chi li ama e da chi li disprezza) o di un fenomeno (mediante il dialogo di due attori coinvolti: per esempio l'effetto serra, visto da un ciclone e da un ghiacciaio).

Al fine di recuperare conoscenze disciplinari, si può rivelare utile anche l'adozione della tecnica dei sei cappelli, pensata dallo psicologo De Bono (2004). Ideata per la risoluzione dei conflitti, è qui declinata in ambito glottodidattico. Agli studenti si può chiedere di formulare commenti riguardo a un certo fenomeno, secondo un registro particolare, contrassegnato da un cappello colorato:

- cappello bianco: giudizio neutrale; ha a che fare con i fatti;

- cappello rosso: sentimenti accesi (odio/amore; mi piace/non mi piace), espressi mediante un giudizio apodittico, che non spiega e non dà ragione alle affermazioni che sostiene;

- cappello giallo: pensiero positivo, ottimista, costruttivo;

- cappello viola: pensiero negativo, pessimista, cupo, ipercritico;

- cappello verde: pensiero creativo; si rilanciano nuove idee, si offrono alternative, si punta al cambiamento;

- cappello blu: $\quad$ si ha una supervisione dei processi, si tiene conto dei bisogni, si tirano le conclusioni.

Si immagini, dunque, di avviare una discussione a gruppi, in una classe CLIL, attorno al tema del letargo degli animali. Gli alunni sviluppano, a coppie o in piccolo gruppi, simultaneamente, frasi attenendosi al colore del cappello di turno:

- cappello bianco: Alcuni animali vanno in letargo in inverno: dormono in una tana e consumano lentamente le energie di grasso accumulate durante l'estate.

- cappello rosso: Gli animali in letargo sono tenerie inoffensivi; gli animali in letargo sono sporchi e puzzano.

- cappello giallo: Un animale in letargo è un buon esempio di risparmio energetico. cappello viola: Un animale in letargo può non essere pronto a reagire in situazioni di pericolo (allagamento o ostruzione della tana). 


\section{- cappello verde: $\quad$ Si potrebbe chiedere agli insegnanti di non darci lezioni durante le vacanze, in modo che le vacanze siano come un letargo. \\ - cappello blu: $\quad$ Andare in letargo ha una funzione di adattamento all'ambiente}

Pure la tecnica delle domande ripetute (presentata in FRANK, RINVOLUCRI, 1991) sollecita una visione più ampia dei fenomeni‘. Nell'esempio a seguire lo studente A ripete alla studente $\mathrm{B}$ una domanda che ha per oggetto la percezione della propria identità:

Chi sei?

Uno studente di italiano

Chi sei?

Guillermo Tovar.

Chi sei?

Un abitante di Barcellona

Chi sei?

Uno spagnolo.

Chi sei?

Un uomo di trentatré anni

Chi sei?

Un tifoso del Barcellona.

In quest'altro caso, la domanda ripetuta da parte di uno studente all'altro punta, invece, a far emergere aspetti meno noti del luogo in cui si vive:

Dove abiti?

$$
\text { In Germania }
$$

Dove abiti?

In una casa del Seicento.

Dove abiti?

In un piccolo paese vicino a Brema.

Dove abiti?

In una via isolata.

\footnotetext{
4 È interessante per l'insegnante, per inciso, analizzare l'ordine con cui vengono date le risposte, in modo da valutare le caratteristiche che hanno $\mathrm{m}$ aggiore salienza per il ragazzo.
} 
Dove abiti?

In un posto immerso nella natura.

Dove abiti?

In un paese che sento molto mio.

2.4. Trasformare l'input

In ambito glottodidattico il pensiero divergente si può manifestare, infine, mediante attività di manipolazione del testo, per esempio:

- L'espansione (si pensi al completamento di un proverbio: tra il dire e il fare c’è di mezzo il mare, e io ho appena imparato a nuotare);

- Una modifica riguardante:

o il genere (dalla lettera informale alla radiocronaca);

o il tema (si pensi ad un testo in cui alcune parole vengono sostituite);

o il registro (si pensi ad una canzone triste trasformata in allegra);

o una parte (la riscrittura del finale);

o una componente grammaticale (di genere e/o di numero delle persone descritte, oppure di tempo verbale);

- Il doppiaggio di una sequenza video, una volta riscritti i dialoghi;

- La composizione di colonne sonore, l'allestimento di improvvisazioni, l'abbozzo di disegni relativi a sequenze tratte dal testo, che possono valere come integrazione del testo.

L'atto del manipolare accorcia le distanze tra studente e lingua straniera, specie laddove il testo viene rappresentato con un'aura di sacralità, come avviene, per esempio, nell'insegnamento della letteratura.

\section{Conclusione}

La divergenza può imprimere all'educazione linguistica una virata: anziché let- 
tera morta, la lingua altra può essere impiegata come grimaldello per accedere a parti di sé altrimenti poco note, per avere una visione più globale dei fenomeni, per ridefinire le relazioni.

Da parte dell'insegnante, la creatività, prima ancora di essere insegnata, viene "agita”, diventa parte di un modus operandi che riconosce un posto d'onore al cambiamento e alla sperimentazione.

\section{Bibliografia}

ALONSO Tapia Jesús. “Knowledgement Assessment and Conceptual Understanding”, in Limón M., Mason L. (eds), Reframing the Processes of Conceptual Change. Dortdrecht: Kluwer, 2000, pp. 389-413.

AZNAR Guy. "Préciser le sens du mot «creativité»", Synergies Europe, 4, 2009, pp. 23-37.

AQUATI A., DE PASCALE C., SCUDERI F., Semini V. La mela di Newton. Torino: Loescher. Vol. B, 2005.

BALBONI Paolo Emilio. Didattica dell'italiano a stranieri. Roma: Bonacci, 1994.

BALBONI Paolo Emilio. Tecniche didattiche per l'educazione linguistica. Torino: Utet, 1998.

BALBONI Paolo Emilio. Modelos operativos para la educación lingüística. Perugia: Guerra, 2007.

BATESON Gregory. Mente e natura, Adelphi, Milano, 1984.

BEVILACQUA Giuseppe. Compendio di educazione alla voce. Roma: Archivio Accademia Nazionale di Arte Drammatica, 2005.

CAON Fabio. Pleasure in Language Learning. Perugia: Guerra, 2007.

CASSANY Daniel. Reparar la escritura. Didáctica de la correcíon de lo escrito, Barcelona: Graó, 1993.

CSIKSZENTMIHALYI Mihaly. The Evolving Self: A Psychology for the Third Millennium, New York: HarperCollins, 1993.

DACEY John S., FIORE Lisa B. Il bambino ansioso. Trento: Erickson, 2002.

D’ANGELO Katia. "La costruzione della conoscenza. Descrizione della Ricostruzione di Conversazione”. Bollettino Dilit, 1, 2006, pp. 22-34.

DE BONO Edward. Una bella mente. Trento: Erickson, 2004.

DELLER Sheelagh, PRICE Christine. Teaching Other Subjects Through English. Oxford: OUP, 2007.

DELMASTRO Ana Lucia. "El andamiaje metacognitivo en contextos de aprendizaje de una lengua estranjera”. Didáctica. Lengua y Literatura [di prossima pubblicazione].

EHRMAN Madeline. "Ego Boundaries and Tolerance of Ambiguity in Second Language Learning”, in Arnold Jane (a cura di), Affect in Language Learning, Oxford: OUP, 1999, pp. 68-85.

FAGIN Larry. The List Poem. New York: Teachers \& Writing Collaborative, 1991.

FERENCICH Roberta, TORRESAN Paolo. Giochi senza frontiere. Firenze: Alma, 2005.

FORAPANI Daniela. .It, Internet nella classe di italiano. [CD-Rom]. Roma: Edilingua, 2001. 
FRANK Christine, RINVOLUCRI Mario. Grammar in Action Again. London: Prentice-Hall, 1991. FREDDI Giovanni. Didattica delle lingue moderne, Bergamo: Minerva Italica, 1979.

GARDNER Howard. Educare al comprendere. Milano: Feltrinelli, 1991.

GRIFFITHS Griff, KEOHANE Kathy. Personalizing Language Learning, Cambridge: CUP, 2000.

HOLMES Vicki L., MOULTON, Margaret R. Writing Simple Poems. Cambridge: CUP, 2001.

MEDNICK Sarnoff. "The Associative Basis of the Creative Process”. Psychological Review, 69, 1962, pp. 220-232. MORGAN John, RINVOLUCRI Mario. Vocabulary. Oxford: OUP, $2004^{2}$.

MULLER Catherine. "La créativité dans des commentaires de photographies en classe de français langue étrangère”, Synergies Europe, 4, 2009, pp. 89-104.

PIAZZOLI Erika. "Metodologia Process Drama e competenza interculturale”. Culturiana 3/4, 2009, pp. 71-75.

PUCHTA Herbert, RINVOLUCRI Mario, ARNOLD Jane. Imagine That! Cambridge: CUP, 2007.

PUGLIESE Chaz. Creativity in Language Teaching. Peaslake: Delta, 2010.

RINVOLUCRI Mario. “The Inevitability of Student Creativity”, Synergies Europe, 4, 2009, pp. 167-172.

SABATANO Claudia. Come si forma la memoria. Roma: Carocci, 2004.

SIMIONATO Maria Assunta. "Tecniche didattiche per lo sviluppo della competenza fonologica”. Bollettino Itals, 14, www.itals.it, 2004.

STEVICK Earl W. Images and Options in the Language Classroom. Cambridge: CUP, 1986.

TORRESAN Paolo. "Risvegliare l'attenzione dello studente: nuove tecniche di comprensione di un testo in lingua straniera”, Synergies Venezuela, 4, 2009a, pp. 11-37; 38-66.

TORRESAN Paolo. "Polirappresentazionalità e circolarità nella didattica dell'italiano". Zeitschrift für Romanische Sprachen und ihre Didaktik, 3, 2009b, pp. 80-89.

TORRESAN Paolo. “Ascoltare con un proposito”, Officina, 4, 12 www.almaedizioni.it, 2010.

VIANELLO Alessandro. Il fotoracconto e il fotoclip, Tesi di Master, Università Ca’ Foscari, Venezia, 2001.

WESSELS Charlyn. Drama. Oxford: OUP, 1987.

WOOD D., BRUNER J. S., ROSS G. "The Role of Tutoring in Problem Solving”, Journal of Child Psychology, 17, 1976. 


\section{Appendice:}

Test di flessibilità cognitiva (tratto da Dacey, Fiore, 2000,pp. 87-89)

Scopo di questa attività è quello di attivare la vostra immaginazione. Scrivete tutte le domande che vi vengono in mente a proposito della figura - ad esempio "Come si chiama il pagliaccio?”. Non limitatevi esclusivamente a domande a cui può essere data risposta solo guardando la figura. Cercate di porre interrogativi che nessun altro penserebbe di fare. Il tempo a disposizione è di cinque minuti. Non continuate a leggere finché non avrete completato questo esercizio.

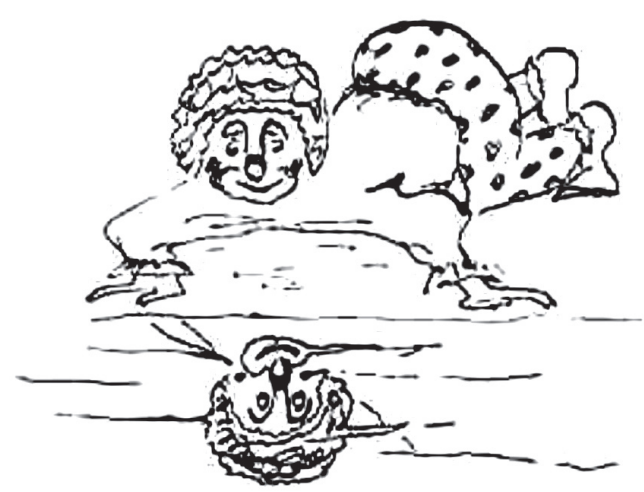

Figura 3. Test di flessibilità (tratta da Dacey, Fiore, 2000, p. 88).

Contate il numero di domande che rientrano chiaramente nelle varie categorie. L'analisi di diverse migliaia di risposte date a questo test ha stabilito che esistono venti categorie di domande, elencate di seguito. Il punteggio massimo è, dunque, venti.

Per esempio domande come "da dove viene?" e "dove li ha presi quei pantaloni?" riceverebbero entrambe un punto. Tuttavia se aveste posto domande del tipo "di che colore ha i capelli?" e "come sono i suoi capelli al tat to?" ricevereste soltanto un punto, perché entrambe rientrano nella categoria 8, "capelli" (vedi lista seguente). Maggiore è la varietà delle vostre domande, più alto sarà il punteggio e, dunque, la valutazione della vostra flessibilità. 


\section{Categorie per l'attribuzione del punteggio nel test di flessibilità}

1. Personaggi che non rientrano nell'immagine (ad esempio, la sorella del pagliaccio)

2. Costume da pagliaccio

3. Elementi etnici, razza, religione, lingua e altro

4. Descrizione di caratteristiche fisiche del personaggio

5. Emozioni, pensiero, personalità del personaggio

6. Famiglia e casa del personaggio

7. Il terreno (superficie)

8. Capelli

9. Luogo, ambientazione e situazione

10. Magia

11. Occupazione e lavoro del personaggio

12. Pantaloni

13. Azioni fisiche riguardanti la superficie che riflette l'immagine

14. Azioni fisiche non riguardanti l'acqua

15. Superficie che riflette l’immagine

16. Camicia

17. Scarpe

18. Orario, età, passato, presente, futuro

19. Sott'acqua

20. Significato globale della scena

Un modo tipico di affrontare questa attività è quello diporre una domande riguardo ai capelli o alle scarpe del pagliaccio, proseguendo con sei o sette altri interrogativi sempre sullo stesso elemento. In questo modo non si contraddicono le istruzioni ma non si è altrettanto fantasiosi di chi pone domande relative a sette diversi aspetti della scena. Le persone che pongono domande su molti diversi aspetti sono considerate flessibili ed è stato dimostrato che sono più fantasiose di quelle che limitano il campo delle proprie domande. 